\title{
Phenotypic diversity of Haemophilus influenzae and Haemophilus parainfluenzae isolates depending on origin and health condition
}

\author{
Sylwia AndrzejCzuK ${ }^{1 \star}$, Edyta ChwiejCZaK ${ }^{2}$, Urszula Kosikowska ${ }^{1}$, \\ Dagmara Stepien-PysniaK ${ }^{3}$, Anna Malm ${ }^{1}$ \\ ${ }^{1}$ Department of Pharmaceutical Microbiology with Laboratory for Microbiological Diagnostics, Medical University of Lublin, Chodzki 1, \\ 20-093 Lublin, Poland \\ ${ }^{2}$ Students Scientific Association at the Department of Pharmaceutical Microbiology with Laboratory for Microbiological Diagnostics, \\ Medical University of Lublin, Chodzki 1, 20-093 Lublin, Poland \\ ${ }^{3}$ Department of Veterinary Prevention and Birds Diseases, Institute of Biological Basis of Animal Diseases, Faculty of Veterinary \\ Medicine, University of Life Sciences in Lublin, Akademicka 12, 20-950 Lublin, Poland
}

\section{ARTICLE INFO}

Received 05 June 2017

Accepted 18 July 2017

\section{Keywords:}

Haemophilus influenzae,

Haemophilus parainfluenzae, microbiota,

phenotypic diversity.

\begin{abstract}
Background. Haemophili are common human microbiota representatives. The aim of our study was to investigate a diversity of Haemophilus spp. isolates selected from clinical specimens on the basis of biochemical characteristics, biotypes distribution, protein profiles and antimicrobial resistance.

Results. A total of 893/1025 (87\%) of haemophili isolates were identified: 260/1025 (25\%) as H. influenzae and 633/1025 (62\%) as H. parainfluenzae. Moreover, a group of 107/1025 (10\%) isolates without species identification (with e.g. abnormal numerical profile) was described as Haemophilus spp. Within the H. influenzae isolates, biotypes II and III were in a great majority (92/893; 10\%, each), whereas among H. parainfluenzae, the most commonly occurring was biotype I and II (301/893, 34\% and 178/893, 20\%, respectively). A similar prevalence of biotypes was obtained regardless of the patient's age or health condition or the type of specimen. A production of beta-lactamases was shown in 46/893 (5\%) haemophili, both $H$. influenzae $(13 / 46,28 \%)$ and $H$. parainfluenzae (33/46, 72\%) isolates.

On the basis of haemophili biochemical characteristics, the cluster analysis using the UPGMA method demonstrated a high degree of phenotypic similarity due to a small distances between isolates taken from both unhealthy children and adults.

Conclusion. Based on biochemical characteristics, about $90 \%$ of haemophili clinical isolates representing human-specific respiratory microbiota were positively identified as $H$. influenzae and $H$. parainfluenzae. The same differences in biotypes and antimicrobial resistance among isolates selected from healthy people or from patients with chronic and recurrent diseases were detected.
\end{abstract}

\section{INTRODUCTION}

Haemophilus influenzae and H. parainfluenzae are an opportunistic and human-restricted microbiota representatives inhabiting the mucous membranes of the respiratory tract. These bacteria colonize the airways of people of all ages, both healthy and with different diseases [10].

The colonization of the respiratory mucous membranes (larynx, trachea, bronchi, lung) by different type of

\begin{tabular}{l}
${ }^{\star}$ Corresponding author \\
e-mail: sylwia.andrzejczuk@umlub.pl \\
\hline
\end{tabular}

microorganisms is a very dynamic process. Under favorable conditions (e.g. treatment or diagnostic procedures, immunodeficiencies), haemophili can damage the adjacent tissue and penetrate into the blood or central nervous system $[18,30]$. Other pathogenic species of these bacteria can colonize the nasopharynx in a transitional period. The bacterial respiratory microbiota composition, both the permanent and transient, is conditioned by the characteristics of the host-individual, as well as by the bacterial mode of life and environment $[30,48]$. 
The first Haemophilus spp. bacteria appear as asymptomatic colonization in the nasopharynx of most children at about three months of age. These include the pathogenic $H$. influenzae species. Children under six months of age rarely are the carriers of these bacteria, because of the presence of maternal antibodies protecting against them [10, $15,40,48]$. However, such passive immunity obtained from the mother's milk does not last long, and $90 \%$ of all children $>1$ year of age are carriers of these bacteria. $H$. influenzae occurs in $3-5 \%$ of all children between 2 and 3 years of age, with the highest degree of colonization being within the respiratory tract mucous membranes. On the contrary, in adults, about $50 \%$ of all individuals are colonized with haemophili $[10,30]$.

Studies from the late $\mathrm{XX}^{\text {th }}$ century described many cases of $H$. influenzae and $H$. parainfluenzae being the etiologic factors of many invasive and non-invasive infections, especially in pediatric patients $[31,52,51,54]$. As members of the HACEK group (Haemophilus spp., Aggregatibacter spp., Cardiobacterium hominis, Eikenella corrodens, and Kingella spp.) [46] or human specific pathogens [5,12], some haemophili may be an important etiologic factors in many local and invasive infections. Both $H$. influenzae and the less common $H$. parainfluenzae can cause pharyngitis and tonsillitis, otitis, sinusitis, epiglottitis, meningitis, bronchitis, sepsis or bacterial endocarditis $[32,45,55]$. They are also frequently identified in chronic obstructive pulmonary disease (COPD) and even in acute septic arthritis or osteoarthritis $[6,9,17,18,21,38,55]$. Currently, H. parainfluenzae is more commonly recognized as an opportunistic pathogen rather than a normal inhabitant [10], especially according to its increased resistance to beta-lactams [11].

A traditional and routinely used biochemical identification of haemophili is essential [35], but in about 1-10\% of cases, the current approaches fail [12,26,42]. However, studies on Haemophilus species have revealed some relatedness between biochemical characteristics, especially with regard to biotypes, and the diseases that have arisen as a consequence of the bacterial colonization.

The aim of this study was to compare the phenotypic diversity of $H$. influenzae and $H$. parainfluenzae as collected from a large group of patients with acute or chronic and recurrent diseases and from healthy people, with regard to the biochemical characteristics, distribution of biotypes and antimicrobials resistance.

\section{MATERIALS AND METHODS}

\section{Bacterial strains}

The study involved a total number of 1025 haemophili isolates taken from various clinical specimens (Table 1), and collected in a museum of the Department of Pharmaceutical Microbiology with Laboratory for Microbiological Diagnostics, at the Medical University of Lublin, Poland. All isolates were divided into two separate groups. The first group included 445 isolates collected from patients comprising of children aged 2-5 years old with upper respiratory tract infections who had undergone adenoidectomy for recurrent pharyngotonsilitis [23]; cystic-fibrosis-positive children aged on average, of 9.97 years [27]; elderly patients with sarcoidosis [24] and with chronic hepatitis C [22], as well as elderly patients with lung cancer [25]. A second group comprising of 580 isolates taken from healthy people (children in the age of 2-13 years old, as well as adults) served as a control group. H. influenzae and H. parainfluenzae, two human-restricted haemophili species with the greatest prevalence and clinical significance were taken into analysis. The research also included four reference strains of Haemophilus spp. from the American Type Culture Collection (ATCC): H. influenzae ATCC 10211, H. parainfluenzae ATCC 33392, H. parainfluenzae ATCC 51505 and additionally H. parainfluenzae ATCC 7901 (recently reclassified into Aggregatibacter aphrophilus) [36].

Table 1. The sources of $H$. influenzae and $H$. parainfluenzae isolates

\begin{tabular}{|c|c|c|}
\hline \multicolumn{2}{|r|}{ The origin of isolates } & No. of isolates \\
\hline \multicolumn{2}{|r|}{ Group of patients } & $n=445$ \\
\hline \multirow{2}{*}{$\begin{array}{l}\text { pediatric patients } \\
(\mathrm{n}=150)\end{array}$} & recurrent respiratory tract infections & 139 \\
\hline & cystic-fibrosis & 11 \\
\hline \multirow{4}{*}{$\begin{array}{l}\text { adult patients } \\
(\mathrm{n}=295)\end{array}$} & upper respiratory tract infections & 27 \\
\hline & lung cancer & 26 \\
\hline & sarcoidosis & 198 \\
\hline & viral hepatitis C & 44 \\
\hline \multicolumn{2}{|r|}{ Group of healthy people } & $n=580$ \\
\hline \multicolumn{2}{|c|}{ children of pre- and primary school age } & 277 \\
\hline \multicolumn{2}{|l|}{ adults } & 303 \\
\hline & Total & 1025 \\
\hline
\end{tabular}

Before performing the research, isolates were frozen at $-70^{\circ} \mathrm{C}$ in trypticase soy broth (TSB, BioRad, USA), with the addition of the Haemophilus Test Medium Supplement (HTMS, Oxoid, UK) and 30\% glycerol (v/v; POCH, Poland). Then, all isolates were cultured on Haemophilus Chocolate Agar (HAEM, bioMérieux, France) and incubated overnight at $35^{\circ} \mathrm{C}$ in an atmosphere of $5 \% \mathrm{CO}_{2}$.

\section{Biochemical identification of isolates}

Biochemical identification was carried out using the commercially available API NH microtests (bioMérieux, France) for Neisseria spp., Haemophilus spp. and Moraxella catarrhalis, and the results were interpreted following the manufacturer's instructions. The composition of API NH strips verified the following 12 biochemical features: the fermentation of the sugars: D-glucose, D-fructose, D-maltose and D-saccharose; the activity of the bacterial enzymes: ornithine decarboxylase, urease, lipase, alkaline phosphatase, $\beta$-galactosidase, proline arylamidase, $\gamma$-glutamyl trasferase and tryptophanase. The results were obtained as a numerical profile, using the tables attached by the manufacturer. This method enabled $H$. influenzae and $H$. parainfluenzae to be categorized into eight biotypes (I-VIII), on the basis of urease (URE) and ornithine decarboxylase (ODC) activity, and the ability of the studied isolates to convert tryptophan into indole (IND), according to the classification of Kilian [20]. Additionally, the isolated bacteria were tested for the presence of penicillinase in a separate microtest (PEN), which was confirmed by the rapid cefinase test (CEF-F; 
bioMérieux, France), in compliance with the Clinical and Laboratory Standards Institute (CLSI) recommendations [7].

Furthermore, for isolates misidentified or representing abnormal numerical profile and unidentifiable in abovementioned method, a presumptive verification by the matrix assisted laser desorption ionization-time of flight mass spectrometry (MALDI-TOF MS) system (Bruker Daltonik, Germany) was performed, according to the modified technique [27]. In this method isolates were identified on the basis of protein specific mass spectra comparing with reference spectra from the integrated database. The following reference strains were included: $H$. influenzae besSt30 THL, H. parainfluenzae 21086307 MLD, H. parainfluenzae DSM 8978 T DSM, $H$. parainfluenzae VP 58646 BOM, $H$. parainfluenzae VP 58974 BOM, and $H$. parainfluenzae VP 59143 BOM. A value indicating correct identification using MALDI TOF MS was $>2.000$. Scores ranging from 2.0002.299 - provided a secure genus identification and probable species identification, and in the range of 2.300-3.000demonstrated high probable species identification [26].

\section{Analysis of phenotypic haemophili diversity}

The biochemical characteristics were presented in a binary system, where 0 and 1 elements were classified as a negative and positive results, respectively. A (0-1)-matrix allowed the evaluation of the similarity matrixes computed via the Dice's coefficient and through phenotypic similarities between isolates. Phenotypic similarity analysis was performed with the data set of $260 \mathrm{H}$. influenzae and $633 \mathrm{H}$. parainfluenzae morphological characters. Four ATCC Haemophilus spp. strains were used as a reference group. The similarity matrix on the basis of the Dice indices (SørensenDice index) allowed a detailed cluster analysis of the various phenotypes by way of utilizing the unweighted pair group method allied with the arithmetic average (UPGMA) method. Phenotypic diversity and the bootstrap analysis of 100 resamplings were calculated using the software package DendroUPGMA tool [14]. Phenograms were constructed using the TreeView 1.6.6 application [39].

\section{RESULTS}

\section{Haemophili isolates identification}

As a result of biochemical and protein profiles detection, a total of $893 / 1025(87 \%)$ of haemophili isolates were positively identified to the species level. The majority of isolates were $H$. influenzae $(260 / 1025,25.4 \%)$ and $H$. parainfluenzae (633/1025, 61.8\%) (Figure 1).

The biochemical identification of 132/1025 (12.9\%) isolates failed, and such bacteria isolates were considered as being either misidentified, representing abnormal numerical profiles or were not identifiable by way of this method. Hence, on the basis of the specific profiles for bacteria proteins, among the non-identified isolates, 7/1025 (0.7\%) were found to not belong to the Haemophilus genus and $18 / 1025$ (1.8\%) were not positively identified at all. These misidentified microorganisms were discarded from further analysis. A group of 107/1025 (10.4\%) isolates was thus recognized by protein profile as other Haemophilus spp., with a score $<2.000$.

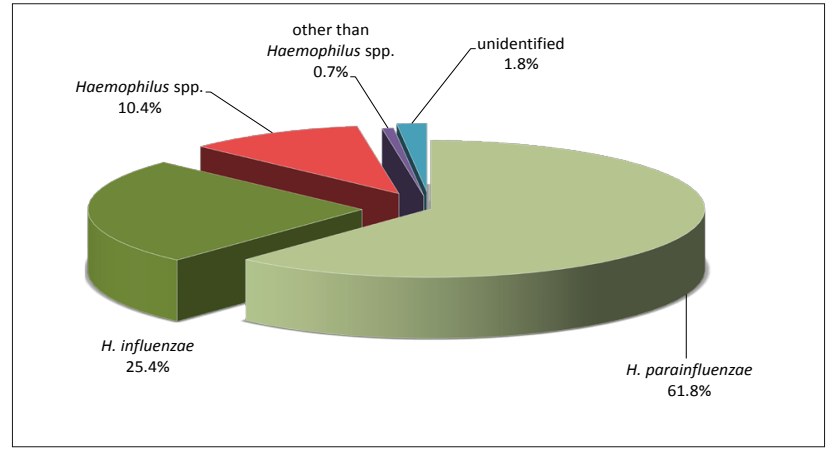

Figure 1. Identification results of bacterial isolates growing on the chocolate agar (HAEM medium) detected on the basis of biochemical characteristics and protein profiles

As regards the diversity of specimens used in our study (Figure 2), it was found that among isolates taken from the nasopharynx, $H$. influenzae was identified with a greater frequency than $H$. parainfluenzae $(99 / 184,53.8 \%$ vs. $85 / 184$, $46.1 \%$ ). However, $H$. parainfluenzae was isolated more frequently within isolates taken from pharyngeal swabs and sputum $(472 / 603,78.2 \%$ and $76 / 102,74.5 \%$, respectively) in comparison to $H$. influenzae $(131 / 603,21.7 \%$ vs. $26 / 102$, $25.4 \%$, respectively). Furthermore, $H$. parainfluenzae was identified the most often through all isolates taken from both children and adults $(214 / 398,53.7 \%$ and 419/495, 84.6\%, respectively), in contrast to $H$. influenzae (184/398, 46.2\% and 76/495, 15.3\%, respectively). Still, H. influenzae isolates were more common whether they were taken from children, both healthy and unhealthy, in comparison to those taken from adult patients. Among isolates taken from healthy children of preschool age, $H$. influenzae was identified with a frequency of 93/177 (52.5\%).

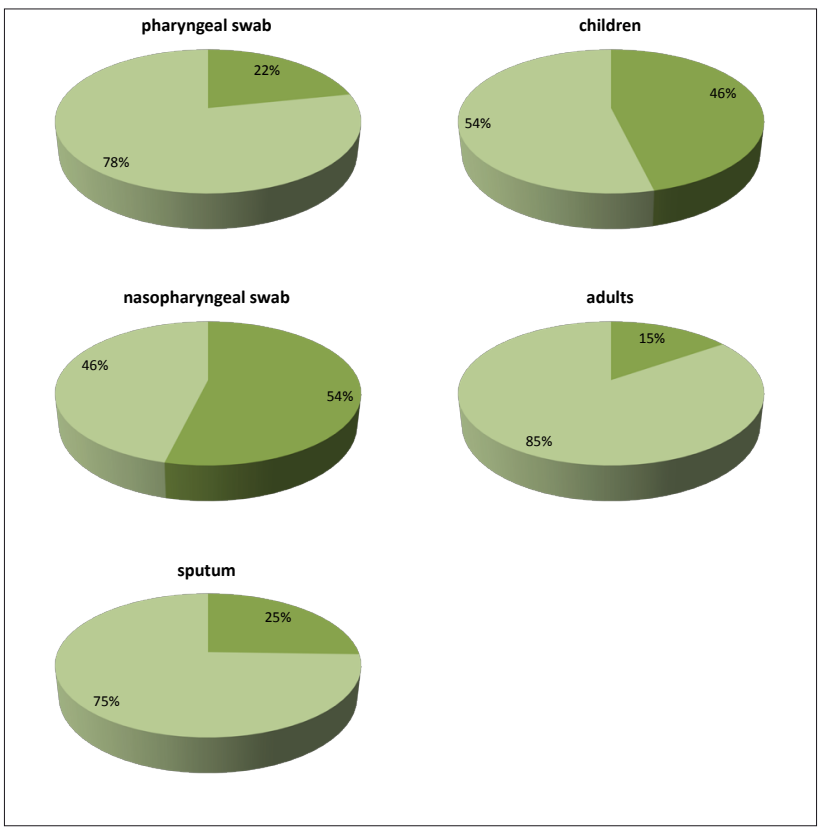

Figure 2. Prevalence of Haemophilus influenzae (dark color) and Haemophilus parainfluenzae (light color) identification in various clinical specimens and groups of people 


\section{Distribution of biochemical characteristics}

A distribution of biochemical characteristics of $H$. influen$z a e$ and $H$. parainfluenzae isolates was analyzed (Figure 3). All H. influenzae isolates showed glucose fermentation, while half revealed fructose fermentation. Other sugars (maltose and saccharose) were used by the studied isolates with a frequency of 15-18/260 (5.7-6.9\%). As regards enzymes activities, no $H$. influenzae isolates possessed the lipase and the proline arylmidase activities. On the other hand, a secretion of urease was strongly dominant in 211/260 $(81.1 \%)$ of the species isolates. As for the indole test, a positive reaction was observed in 135/260 (51.9\%) H. influenzae strains.

The test of biochemical characteristics of $H$. parainfluenzae revealed that all isolates used glucose and most showed the fermentation of other sugars (fructose, maltose, saccharose) with a frequency of 583-623/633 (92.1-98.4\%). The urease- and $\beta$-galactosidase-positives were 293/633 (46.2\%) and 350/633 (55.2\%) of all H. parainfluenzae isolates, respectively. Lipase and proline arylmidase were not disclosed in any of the $H$. parainfluenzae.

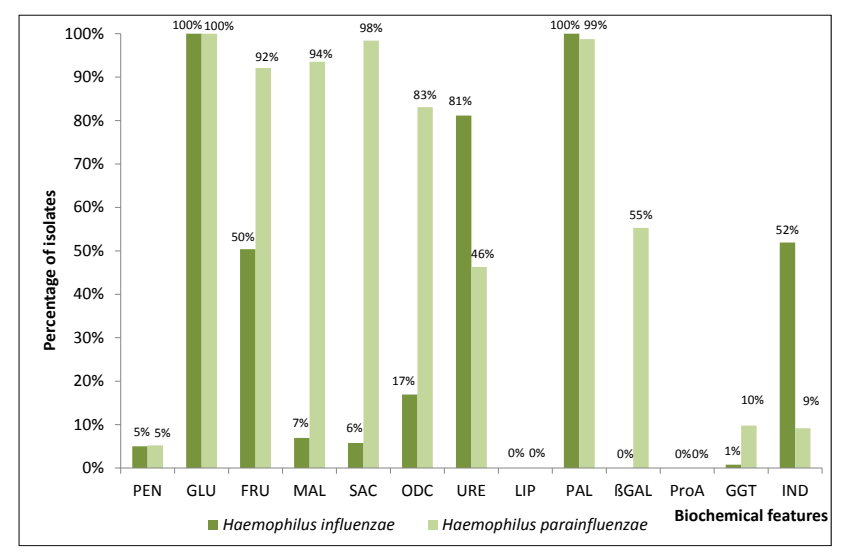

Figure 3. Biochemical characteristics of Haemophilus influenzae $(\mathrm{n}=260)$ and Haemophilus parainfluenzae $(\mathrm{n}=633)$ isolates

\section{Distribution of haemophili biotypes}

As shown in Table 2, within H. influenzae isolates, biotype II and III were dominant (92/893, 10.3\%). These biotypes of $H$. influenzae were dominant both among children and elderly people, independently to health conditions. Of note: an eight times higher frequency of $H$. influenzae biotype I was prevalent in unhealthy pediatric patients, compare to healthy children. Biotype II was dominant in healthy children and in adults independently to health conditions. The studied $H$. parainfluenzae isolates mostly belonged to biotype I and II (301/893, 33.7\% and $178 / 893,19.9 \%$, respectively). Biotype I of $H$. parainfluenzae was the most frequent among healthy children and unhealthy adults (Table 2).

A distribution of haemophili biotypes was analyzed closely among isolates selected from pharyngeal swabs taken from pediatric patients and healthy children (Figure 4). Herein, a low diversity of $H$. influenzae biotypes (mainly II and III biotype) was observed in pharyngeal isolates taken from pediatric patients. Additionally, biotypes I, V, VI and VIII of this species were not detected at all. The biggest diversity of $H$. influenzae biotypes, as well as biotype II and III domination and the absence of biotypes V and VI, was seen among pharyngeal isolates from healthy children.

$H$. parainfluenzae commonly occurred in pharyngeal isolates from both pediatric patients and healthy children (Figure 4). Biotypes I and II, followed by biotypes III and IV, were in a majority, especially in isolates taken both from pediatric patients and in a much greater number of healthy children. Furthermore, among isolates from pediatric patients, biotypes V-VIII were missing.

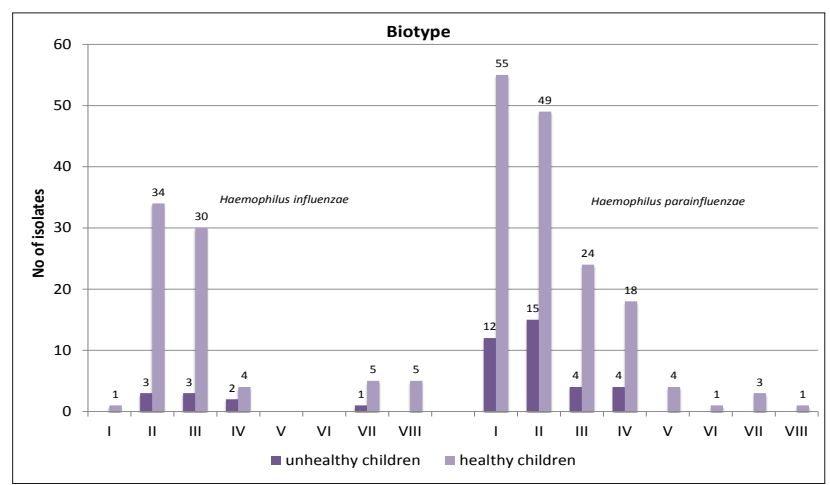

Figure 4. Distribution of haemophili biotypes among pharyngeal Haemophilus influenzae and Haemophilus parainfluenzae isolates taken from pediatric patients and healthy children

Table 2. Distribution of Haemophilus influenzae and Haemophilus parainfluenzae biotypes among isolates taken from children and adults

\begin{tabular}{|c|c|c|c|c|c|c|c|c|c|c|c|c|c|c|c|c|c|c|}
\hline & & & \multicolumn{16}{|c|}{ No. of isolates } \\
\hline \multirow{2}{*}{\multicolumn{3}{|c|}{$\begin{array}{l}\text { Species } \\
\text { Biotype }\end{array}$}} & \multicolumn{8}{|c|}{ H. influenzae $(\mathbf{n}=\mathbf{2 6 0})$} & \multicolumn{8}{|c|}{ H. parainfluenzae $(n=633)$} \\
\hline & & & I & II & III & IV & V & VI & VII & VIII & I & II & III & IV & V & VI & VII & VIII \\
\hline \multirow{3}{*}{\multicolumn{2}{|c|}{$\begin{array}{l}\text { Expected } \\
\text { reaction }\end{array}$}} & ODC & + & - & - & + & + & + & - & - & + & + & - & + & - & + & - & - \\
\hline & & URE & + & + & + & + & - & - & - & - & - & + & + & + & - & - & + & - \\
\hline & & IND & + & + & - & - & + & - & + & - & - & - & - & + & - & + & - & + \\
\hline \multirow{4}{*}{ 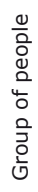 } & \multirow{2}{*}{ 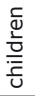 } & unhealthy & 19 & 27 & 25 & 3 & 8 & - & 4 & - & 19 & 22 & 9 & 6 & - & 1 & - & - \\
\hline & & healthy & 2 & 42 & 36 & 4 & 4 & - & 5 & 5 & 55 & 49 & 24 & 19 & 4 & 2 & 3 & 1 \\
\hline & \multirow{2}{*}{$\frac{\stackrel{n}{5}}{\frac{ \pm}{7}}$} & unhealthy & - & 13 & 18 & - & - & - & - & 10 & 132 & 52 & 16 & 5 & 1 & 4 & 2 & - \\
\hline & & healthy & 2 & 10 & 13 & - & 1 & 1 & 1 & 7 & 95 & 55 & 24 & 6 & 18 & 4 & 1 & 4 \\
\hline \multicolumn{3}{|c|}{ Total } & 23 & 92 & 92 & 7 & 13 & 1 & 10 & 22 & 301 & 178 & 73 & 36 & 23 & 11 & 6 & 5 \\
\hline
\end{tabular}

Abbreviations: IND - indole, ODC - ornithine decarboxylase, URE - urease 
A frequency of $H$. influenzae and $H$. parainfluenzae biotypes occurrence among haemophili isolates taken from healthy and unhealthy children and adults is presented in Table 3.

Among both the healthy and unhealthy, $H$. influenzae isolates classified as biotype II (52/260, 20.0\% and 40/260, $15.3 \%$, respectively) and III (49/260, $18.8 \%$ and $43 / 260$, $16.5 \%$, respectively) were dominant (Table 3). Among the isolates taken from sputum, biotype III, followed by biotype VIII, occurred the most $(12 / 35,34.3 \%$ vs. $8 / 35$, $22.9 \%$, respectively). Many isolates were observed in the nasopharyngeal swabs, with the most popular being biotypes II and III (24/86, 27.9\% and 22/86, 25.5\%, respectively).

Among the healthy and unhealthy, $H$. parainfluen$z a e$ isolates classified as biotype I (150/633, 23.6\% and $151 / 633,23.8 \%$, respectively) and II (104/633, $16.4 \%$ and $74 / 633,11.6 \%$, respectively) were dominant (Table 3 ). These biotypes were the most frequent among isolates taken both from healthy children and adults. As revealed in Table 3, $143 \mathrm{H}$. parainfluenzae isolates taken from patients with sarcoidosis were assigned into at least 3 biotypes. Among $63 / 143$ isolates taken from the nasopharynx and 72/143 isolates taken from sputum, biotypes I $(35 / 63,55.5 \%$ vs. $17 / 63,26.9 \%)$ and II $(53 / 72,73.6 \%$ vs. $16 / 72,22.2 \%)$ were dominant. What is more, biotype I was very common in pharyngeal $H$. parainfluenzae isolates taken from patients with viral hepatits C $(28 / 37,75.7 \%)$, while biotype II was predominant among isolates taken from the throat $(22 / 51$, $43.1 \%)$ and nasopharyngeal swabs $(7 / 20,35.0 \%)$ of people with respiratory tract infections. Other biotypes occurred much less frequently.

\section{Beta-lactamase production}

As is shown in Figure 5, in a total of $893 \mathrm{H}$. influenzae and $H$. parainfluenzae isolates, the production of beta-lactamase was detected in 46/893 (5.2\%) isolates, using Pen and cefinase tests. This phenotype of antibiotic resistance was observed in 13/46 (28.3\%) H. influenzae and 33/46 (71.7\%) H. parainfluenzae isolates.

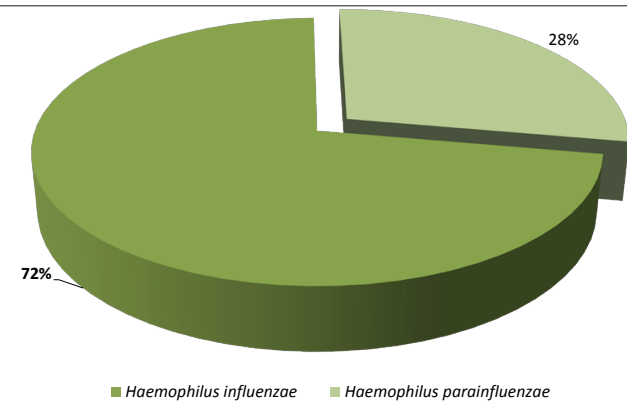

Figure 5. Prevalence of beta-lactamase-positive Haemophilus influenzae and Haemophilus parainfluenzae isolates

The production of beta-lactamases was analyzed through $H$. influenzae and $H$. parainfluenzae isolates taken from both adults and children, whether healthy or unhealthy (Figure 6). H. influenzae beta-lactamase producers included 6/13 $(46.2 \%)$ isolates taken from children with respiratory infections and 1/13 (7.7\%) isolate selected from an adult patient with respiratory tract infection. As is shown in Figure 6, 25/33 (75.7\%) H. parainfluenzae beta-lactamase-positive isolates were drawn from adults, and 8/22 (24.2\%) were obtained from children.

Table 3. Biotyping results within isolates of Haemophilus influenzae and Haemophilus parainfluenzae

\begin{tabular}{|c|c|c|c|c|c|c|c|c|c|c|c|c|c|}
\hline \multirow{4}{*}{$\begin{array}{l}\mathscr{N} \\
\frac{U}{\mathscr{D}} \\
\stackrel{๊}{n}\end{array}$} & \multirow{4}{*}{$\begin{array}{l}0 \\
\frac{0}{\lambda} \\
\frac{0}{0} \\
0\end{array}$} & \multicolumn{3}{|c|}{$\begin{array}{l}\text { Isolates selected from healthy } \\
\text { people }\end{array}$} & \multicolumn{9}{|c|}{ Isolates selected from patients with } \\
\hline & & \begin{tabular}{|c|} 
children \\
in preschool \\
age
\end{tabular} & $\begin{array}{l}\text { children } \\
\text { in school } \\
\text { age }\end{array}$ & adults & \multicolumn{3}{|c|}{$\begin{array}{c}\text { sarcoidosis } \\
\quad(n=178)\end{array}$} & $\begin{array}{l}\text { lung } \\
\text { cancer } \\
(n=15)\end{array}$ & \begin{tabular}{|c|} 
viral \\
hepatitis C \\
$(\mathrm{n}=37)$
\end{tabular} & \multicolumn{2}{|c|}{$\begin{array}{c}\text { cystic } \\
\text { fibrosis } \\
(n=9)\end{array}$} & \multicolumn{2}{|c|}{$\begin{array}{c}\text { other upper } \\
\text { respiratory tract } \\
\text { infections }(n=157)\end{array}$} \\
\hline & & \multicolumn{12}{|c|}{ No. $(\%)$ of isolates } \\
\hline & & \multicolumn{3}{|c|}{ throat } & nasopharynx & throat & sputum & throat & throat & throat & sputum & throat & nasopharynx \\
\hline \multirow{9}{*}{ 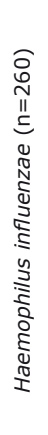 } & & $n=93$ & $n=5$ & $\mathrm{n}=35$ & $\mathrm{n}=8$ & $n=2$ & $\mathrm{n}=25$ & $n=3$ & $\mathrm{n}=0$ & $\mathrm{n}=2$ & $\mathrm{n}=1$ & $\mathrm{n}=10$ & $\mathrm{n}=76$ \\
\hline & I & $2(2.2)$ & - & $2(5.7)$ & - & - & - & - & - & - & - & - & $19(25.0)$ \\
\hline & II & $40(43.0)$ & $2(40.0)$ & $10(28.6)$ & $2(25.0)$ & $2(100)$ & $5(20.0)$ & $2(66.7)$ & - & $1(50.0)$ & - & $4(40.0)$ & $24(31.6)$ \\
\hline & III & $33(35.5)$ & $3(60.0)$ & $13(37.1)$ & $4(50.0)$ & - & $12(48.0)$ & $1(33.3)$ & - & - & - & $4(40.0)$ & $22(28.9)$ \\
\hline & IV & $4(4.3)$ & - & - & - & - & - & - & - & $1(50.0)$ & $1(100)$ & $1(10.0)$ & - \\
\hline & V & $4(4.3)$ & - & $1(2.9)$ & - & - & - & - & - & - & - & - & $8(10.5)$ \\
\hline & VI & - & - & $1(2.9)$ & - & - & - & - & - & - & - & - & - \\
\hline & VII & $5(5.4)$ & - & $1(2.9)$ & - & - & - & - & - & - & - & $1(10.0)$ & $3(3.8)$ \\
\hline & VIII & $5(5.4)$ & - & $7(20.0)$ & 2 & - & $8(32.0)$ & - & - & - & - & - & - \\
\hline \multirow{9}{*}{ 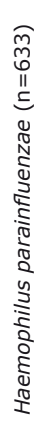 } & & $n=84$ & $n=73$ & $n=207$ & $n=63$ & $n=8$ & $n=72$ & $n=12$ & $n=37$ & $n=4$ & $n=2$ & $n=51$ & $n=20$ \\
\hline & I & $23(27.4)$ & $32(43.8)$ & 95 (45.9) & $35(55.5)$ & $4(50.0)$ & $53(73.6)$ & $6(50.0)$ & $28(75.7)$ & $2(50.0)$ & $2(100)$ & $16(31.4)$ & $5(25.0)$ \\
\hline & II & $26(30.9)$ & $23(31.5)$ & $55(26.6)$ & $17(26.9)$ & $2(25.0)$ & $16(22.2)$ & $3(25.0)$ & $6(16.2)$ & $1(25.0)$ & - & $22(43.1)$ & $7(35.0)$ \\
\hline & III & $17(20.2)$ & $7(9.6)$ & $24(11.6)$ & $8(12.7)$ & $2(25.0)$ & $2(2.8)$ & $1(8.3)$ & - & $1(25.0)$ & - & $6(11.8)$ & $5(25.0)$ \\
\hline & IV & $9(10.7)$ & $10(13.7)$ & $6(2.9)$ & $1(1.6)$ & - & - & $1(8.3)$ & $3(8.1)$ & - & - & $4(7.8)$ & $2(10.0)$ \\
\hline & V & $3(3.6)$ & $1(1.4)$ & $18(8.7)$ & - & - & - & - & - & - & - & $1(1.96)$ & - \\
\hline & VI & $2(2.4)$ & - & $4(1.9)$ & $2(3.2)$ & - & - & $1(8.3)$ & - & - & - & $1(1.96)$ & $1(5.0)$ \\
\hline & VII & $3(3.6)$ & - & $1(0.5)$ & - & - & $1(1.4)$ & - & - & - & - & $1(1.96)$ & - \\
\hline & VIII & $1(1.2)$ & - & $4(1.9)$ & - & - & - & - & - & - & - & - & - \\
\hline
\end{tabular}




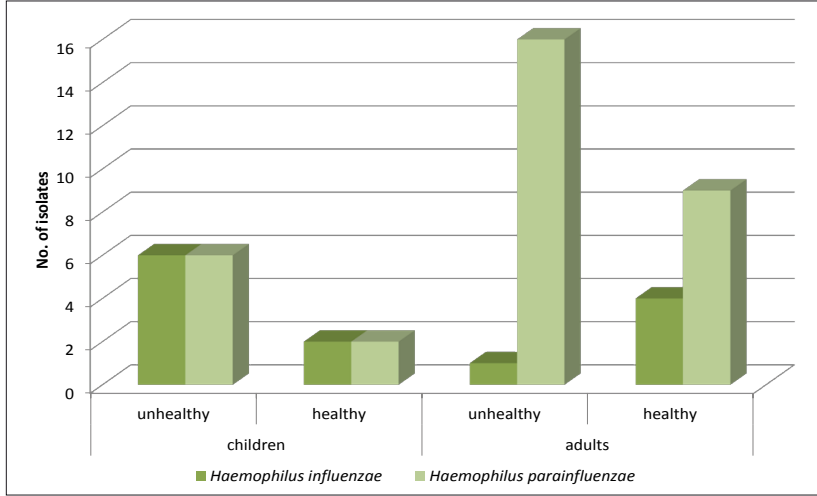

Figure 6. Distribution of beta-lactamase positive Haemophilus influenzae and Haemophilus parainfluenzae isolates taken from children and adults

\subsection{Phenotypic diversity between isolates based on biochemical characteristics}

On the basis of biochemical characteristics, cluster analysis using the UPGMA method was performed for selected isolates. Four ATCC Haemophilus spp. strains were used as a reference group, and two phenograms were constructed. These reveal the diversity of $H$. influenzae and $H$. parainfluenzae phenotypes among isolates taken from adults with sarcoidosis and children with recurrent respiratory tract infections. As shown in Figures 7 and 8, two separate clusters were distinguished: the first of $H$. influenzae isolates and the second of $H$. parainfluenzae. Both clusters were divided further into at least 3 different groups. The Dice coefficient was used to compare a set of biochemical characters, and this revealed H. influenzae ATCC 10211 clustered

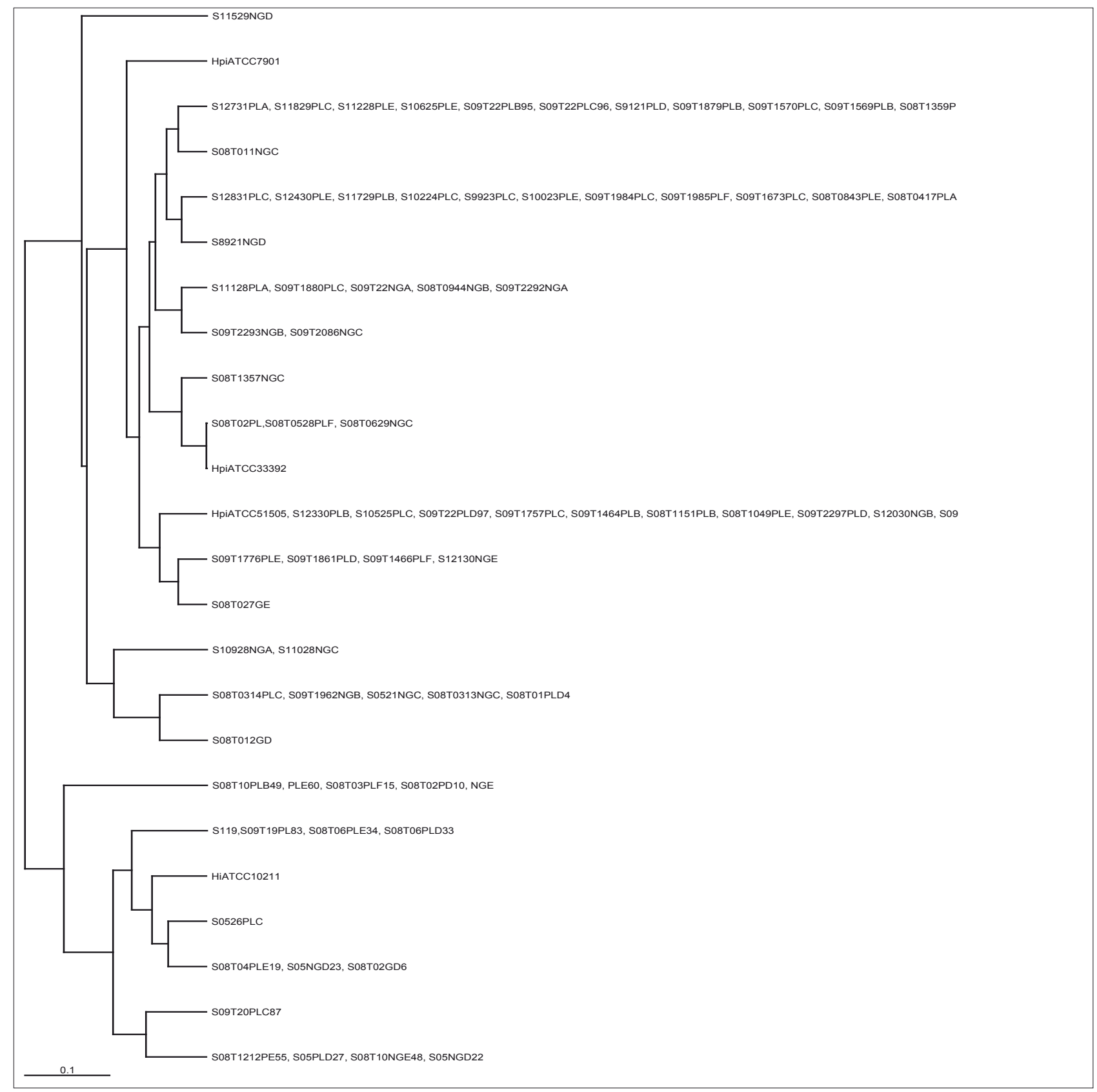

Abbreviations:

$\mathrm{Hpi}$ - Haemophilus parainfluenzae; Hi - Haemophilus influenzae

Figure 7. Phenogram of Haemophilus influenzae isolates discerned through applying the Dice coefficient on biochemical identification results 
within other $H$. influenzae isolates taken from patients, as well as three $H$. parainfluenzae reference strains (ATCC 7901, ATCC 33392, ATCC 51505) within other H. parainfluenzae isolates.

\section{DISCUSSION}

During our study, a diversity of 1025 Haemophilus spp. isolates taken from people either of a different age or of diverse health conditions was determined using biochemical characteristics and antimicrobial phenotypes. Our work revealed that among isolates derived both from children and adults, $H$. parainfluenzae was identified with the greatest frequency. Accordingly, of the $87 \%$ of all isolates which were positively identified using the biochemical method, $25 \%$ of the total were $H$. influenzae and $62 \%$ of the total were
H. parainfluenzae. However, a routinely used culture-dependent biochemical identification test of haemophili failed in $13 \%$ of all cases, especially where isolates representing abnormal numerical profiles were misidentified. Of note: some isolates were not identifiable at all by this method.

Of the positively identified, $H$. influenzae was characterized more frequently among isolates taken from nasopharyngeal swabs and it was more common in specimens taken from children, whether healthy (especially when of preschool age) or unhealthy, in comparison to those taken from adults. In contrast, $H$. parainfluenzae was significantly more common within isolates taken from pharyngeal (e.g. both from healthy and unhealthy children) and sputum samples. A frequency of $H$. parainfluenzae increased between isolates taken from children in preschool age, through isolates

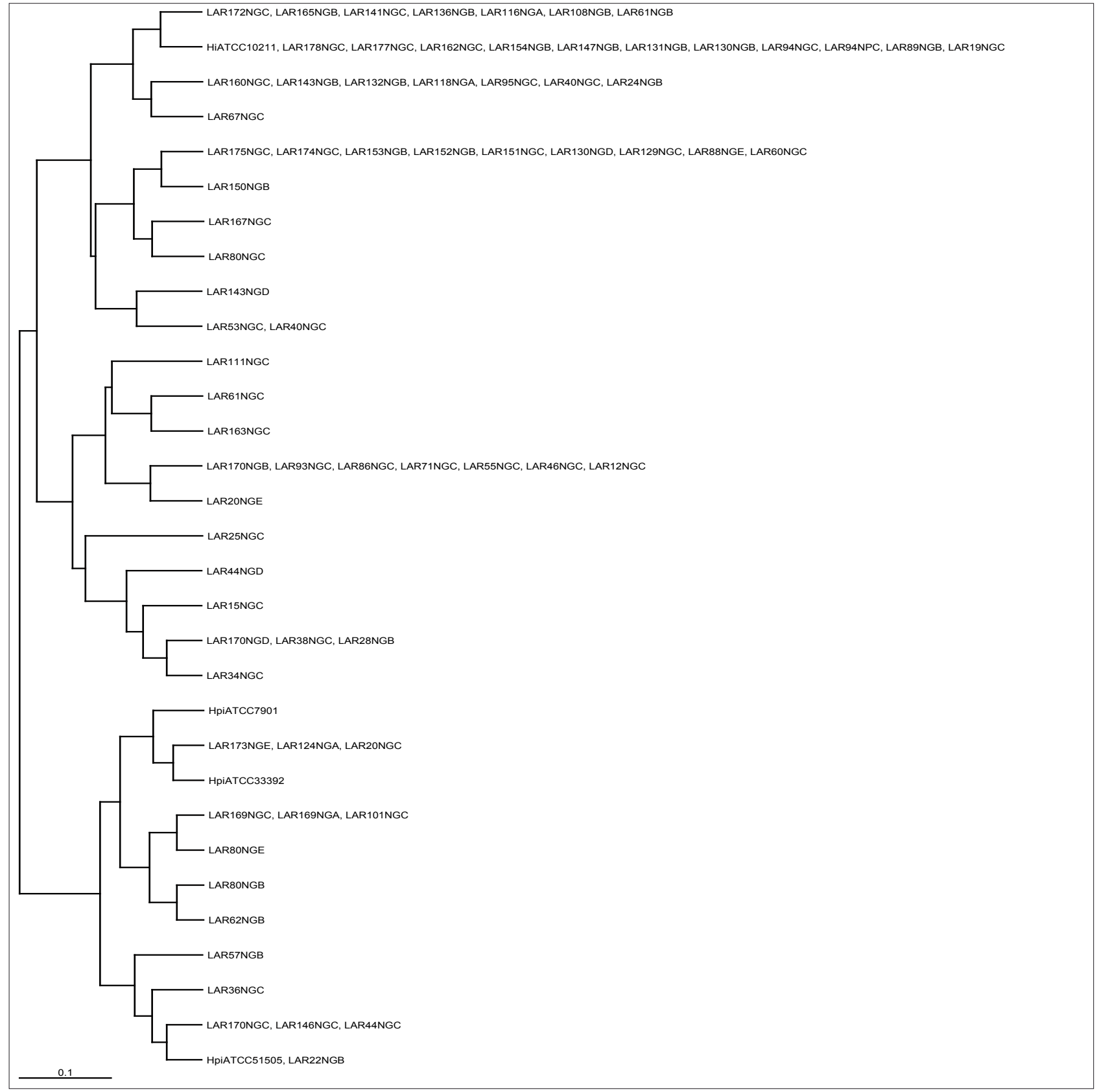

Abbreviations:

$\mathrm{Hpi}$ - Haemophilus parainfluenzae; Hi - Haemophilus influenzae

Figure 8. Phenogram of Haemophilus parainfluenzae isolates revealed through applying the Dice coefficient on biochemical identification results 
obtained from school age children, to become the highest proportionally in the group of isolates taken from adults.

To detect whether the employed biochemical identification method was correct and reliable, two phenograms were generated for selected $H$. influenzae and $H$. parainfluenzae isolates obtained from adults with sarcoidosis and from children with upper respiratory tract infections. In both phenograms, two separate clusters of $H$. influenzae and $H$. parainfluenzae could be distinguished. These were divided further into at least 3 different groups. All clusters were characterized by their high diversity. Our cluster analysis confirmed that the biochemical identification was made properly and that it correctly defined the species of haemophili isolates.

Many authors claim that biochemical identification is an accurate and reliable method for the clinically routine identification of haemophili, for the biotyping of Haemophilus spp. and for the detection of beta-lactamasesproducing isolates $[2,33]$. Our results are in accordance to others which put forward that biochemical methods usually failed in $1-10 \%$ of cases [12]. In spite of the compatibility of our biochemical characterization with a supplied table of positive reactions, to verify and exclude some uncertain results, the MALDI-TOF MS method was also used. This is not a routinely applied technique in bacterial diagnostics. Nevertheless, it had crucial advantages: allowing identification directly from culture plates within minutes, being procedurally simple, having low-sample volume requirements and needing no expensive reagents or high accuracy. Literature states that the MALDI-TOF MS can be a useful tool in the diagnostics of microorganisms rarely isolated or found problematic in routine diagnostics [26,28]. Moreover, it has been successfully adapted to Pasteurellaceae and Haemophilus spp. identification [5,8,13,29].

Our results are consistent with literature reports stating that $H$. parainfluenzae is a significant pathogen associated with various invasive infections $[2,11,40,41,52]$. Moreover, in our work, a strong predominance of $H$. influenzae in isolates taken from pharyngeal swabs of healthy children was observed (with approximately 76-89\% frequency), while H. parainfluenzae occurred more rarely [31,52]. However, in nasal samples from healthy kindergarten children in China, H. influenzae was a less common pathogen [40].

According to our results, both $H$. influenzae and $H$. parainfluenzae showed similar saccharides fermentation abilities. Still, glucose and fructose fermentation were observed as basic carbon sources in all strains. Other sugars (fructose, maltose, saccharose) were used more frequently by H. parainfluenzae.

A strongly distinguishing characteristic is that both haemophili species vary in enzyme activity. About $70 \%$ of all $H$. parainfluenzae and all $H$. influenzae isolates secreted the alkaline phosphatase, while a secretion of urease was strongly dominant in $H$. influenzae. In addition, ornithine decarboxylase activity was observed in the most $H$. parainfluenzae isolates, and $\beta$-galactosidase activity was disclosed in $39 \%$ of all $H$. parainfluenzae, while $H$. influenzae strains were not able synthesize this.

The relationship between biotype and origin (type of diagnostic material) of isolates were also examined in this study. In general, a significant predominance of biotypes II-III in $H$. influenzae was identified from taken isolates, regardless of the patient's age, type of specimen and health status. Among $H$. influenzae isolates obtained from the sputum of patients with sarcoidosis, biotype III and VIII occurred the most frequently.

Our results were in agreement with publications stating a dominance of biotype I over biotype II among $H$. influenzae strains $[2,33,54]$. In our work, biotyping according to a scheme devised by Kilian [19] resulted in seeing the predominance of $H$. influenzae biotype I, followed by biotypes II and III, respectively [2]. Recently, a distinct predominance of $H$. influenzae biotypes I and II was also observed by Uraz et al. [52]. There were, as well, various prior-mentioned studies noting a predominance of $H$. influenzae biotype I in situations of acute infection, such as salpingitis or pneumonia. This may be due to the biotype holding a specific affinity to the ciliated epithelium cells $[43,47]$. This biotype was also sometimes seen as being associated with severe meningitis or cystic fibrosis, in children $[1,44]$, and, generally, with other invasive infection situations [16] induced by H. influenzae (such as sepsis or meningitidis) [54].

Our study was also in agreement with a study involving $H$. influenzae isolates collected from patients with cystic fibrosis. Herein, biotype I dominated with $>80 \%$ frequency, whereas in a group of patients with respiratory infections and in a control group, biotype II was the most common [54]. Still, different results were obtained by Munson and Doern [33]. In their study, $208 \mathrm{H}$. influenzae isolates were collected from people with haemophili-dependent infections, and most were biotype II, followed by biotypes III and I. Other researchers put forward that upper respiratory tractassociated $H$. influenzae isolates usually belong to biotypes II and III and are a part of normal throat microbiome, or, at the same time, are an etiologic factor of a variable number of infections e.g.: sinusitis, otitis media, acute and chronic exacerbations of lower respiratory tract infection, as well as acute or chronic conjunctivitis [1, 34, 47]. In the most described cases of Haemophilus spp.-induced infections, $H$. influenzae biotype II was the most frequent, and most of the non-typeable $H$. influenzae (NTHi) isolates belonged into biotypes II-VI. In turn, $H$. influenzae biotype V was commonly isolated from ear infections [47]. Other biotypes were less common, however, a strong predominance of one of the Haemophilus spp. biotypes is probably still geographically-dependent [49]. Additionally, it is suggested that the presence of the NTHi biotype IV could be related to mother-infant complicated genital infections. This is because $H$. influenzae and $H$. parainfluenzae were thought to present specific morphology and protein patterns (a peritrichous fimbriation and a very peculiar homogeneous unique outer membrane pattern) [53]. However, in further work, this notion has been discarded, as no such a correlation was found [16].

In our study, we saw that $H$. parainfluenzae biotypes I-II were dominant among isolates taken from healthy and unhealthy people regardless of the age of these persons. This result is in agreement with many earlier reports $[37,50,30,40]$. Furthermore, we observed a greater $H$. parainfluenzae biotypes diversity (I-VIII) within isolates taken 
from pharyngeal specimens. Moreover, among pharyngeal isolates taken from patients with sarcoidosis, biotype II dominated.

In contrast to our studies, a strong predominance of biotype III $H$. parainfluenzae isolates was observed by Uraz et al. [52]. According to their results, about $13-15 \%$ of all tested $H$. parainfluenzae isolates belonged to biotypes $\mathrm{V}$ and III, while other biotypes were represented by at least 5 isolates [33]. Other researchers reported H. parainfluenzae biotype I isolates in sputum samples taken from adult patients $>50$ years old, as well as from children $<1$ year of age [37]. What is more, biotypes II and III were identified more frequently in persons $1-5$ years old and from $>20$ years of age [37], and are considered by some researchers as the possible causal agents of continuous ambulatory peritoneal dialysis (CAPD) peritonitis [3].

Biotype II is also reported as a cause of acute episodes of bronchitis, while biotype III brings about chronic bronchitis [50]. Biotypes III, IV, VI- VIII are still observed less frequently. Through identified as $H$. influenzae and $H$. parainfluenzae isolates, biotypes VI-VIII appeared very rarely. This confirms actual biotypes prevalence, yet it is in disagreement with a study conducted on patients with various Haemophilus spp. infections. In this, both $H$. influenzae and $H$. parainfluenzae were isolated in a similar way [33].

Our results showed that about $5 \%$ of all haemophili isolates (both $H$. influenzae and $H$. parainfluenzae) are able to produce a beta-lactamases. In our work, we saw that betalactamase-positive haemophili occurred more frequently among isolates taken from adults than from children. Furthermore, the majority of beta-lactamase-producing $H$. parainfluenzae strains were isolated from unhealthy adults (e.g. from patients with sarcoidosis, viral hepatitis $\mathrm{C}$ or upper respiratory tract infections). Moreover, $H$. influenzae betalactamase producers included the isolates from children with respiratory infections and the isolate from an adult person with a respiratory tract disease.

The data presented in our study are in general agreement with reports from the 1980's wherein beta-lactamasepositive $H$. influenzae and $H$. parainfluenzae were identified with a similar frequency of about 5.6-9.5\% [31,51]. The increasing emergence and spread of haemophili resistance has become a distinct problem [10]. The primary mechanism of $H$. influenzae resistance to beta-lactam antibiotics is the production of beta-lactamases. This mechanism results in a resistance to aminopenicillins (ampicillin, amoxicillin) without a loss of the sensitivity for combination inhibitor therapy (amoxicillin-clavulanic acid) [10,11]. The prevalence of beta-lactamase-positive strains isolated from respiratory tract infections and asymptomatic colonizers vary from a few to several tens of percent in the various European countries $[10,11,15,48]$.

A strong relationship between beta-lactamase production and biotype has also been repeatedly observed, and in determining beta-lactamase production, our results were mostly in agreement with the cefinase test for Haemophilus spp. strains [2]. Still, while biotype I is most commonly found in $H$. influenzae isolates, the majority of beta-lactamase positive isolates are of biotype $\mathrm{V}$ isolates [54]. However, strong differences in beta-lactamase production has been noted among $H$. parainfluenzae isolates, and such mechanism of antimicrobial resistance has been seen as more common in biotype I and II isolates [54]. Indeed, beta-lactamase-positive $H$. parainfluenzae isolates were identified twice more than from $H$. influenzae. This situation confirms the hypothesis that $H$. parainfluenzae is a reservoir of resistance genes within the Haemophilus genus [10,11,51].

In summary, In our study, a great diversity of groups of Haemophilus spp. isolates from various clinical specimens were determined and analyzed. The obtained data has led us to conclude that the derived biochemical features and biotyping of human-restricted haemophili isolates were performed properly on the basis of the chosen biochemical method. In our study, H. influenzae and H. parainfluenzae were positively identified and isolated the most frequently. The data generated has revealed that both Haemophilus species have evolved, as distinct qualitative changes in biotypes distribution were observed. Furthermore, our study shows that biotype II and III predominated within $H$. influenzae isolates, while, in contrast, biotypes I and II predominated within $H$. parainfluenzae isolates. Such trend was found regardless of the patient's age, health status or type of specimen. Additionally, in 5\% of all obtained isolates of both species of haemophili, the production of beta-lactamases was observed. On the basis of literature data and our study results, we conclude that the strong prevalence of $H$. parainfluenzae needs to be monitored due to its high and still increasing prevalence, its distinct changes in biotype distribution, its strong morphological and biochemical similarity to $H$. influenzae and its possibility of being a reservoir of resistance genes.

\section{REFERENCES}

1. Alrawi, A. M. et al.: Biotypes and serotypes of Haemophilus influenzae ocular isolates. Br. J. Ophthalmol., 86, 3, 2002.

2. Barbé G. et al.: Evaluation of API NH, a new 2-hour system for identification of Neisseria and Haemophilus species and Moraxella catarrhalis in a routine clinical laboratory. J. Clin. Microbiol., 1, 32, 1994.

3. Betriu C. et al..: Peritonitis caused by Haemophilus parainfluenzae in a patient undergoing continuous ambulatory peritoneal dialysis. J. Clin. Microbiol., 37, 9, 1999.

4. Bogaert D. et al.: Variability and diversity of nasopharyngeal microbiota in children: a metagenomic analysis. PLOS One, e17035, 6, 2011.

5. Bruin J. P. et al.: Identification of Haemophilus influenzae and Haemophilus haemolyticus by matrix-assisted laser desorption ionization-time of flight mass spectrometry. Eur. J. Clin. Microbiol. Infect. Dis., 33, 2014.

6. Christou L. et al.: Acute Haemophilus parainfluenzae endocarditis: a case report. J. Med. Case Rep., 3, 2009.

7. Clinical and Laboratory Standards Institute (CLSI). Performance Standards for Antimicrobial Susceptibility Testing; Twenty-Fifth Informational Supplement; M100-S25. Clinical and Laboratory Standards Institute, Wayne, PA, 2015.

8. Couturier M. R. et al.: Identification of HACEK clinical isolates by matrix-assisted laser desorption ionization-time of flight mass spectrometry. J. Clin. Microbiol., 49, 2011.

9. Cunha B. A., Brahmbhatt K., Raza M.: Haemophilus parainfluenzae aortic prosthetic valve endocarditis (PVE) successfully treated with oral levofloxacin. Heart Lung, 4, 44, 2015.

10. Ebbing R., Robertson C. F., Robinson P.J.: Haemophilus influenzae and Haemophilus parainfluenzae in cystic fibrosis: 15 years experience. J. Med. Microbiol. Diag., 5, 2015. 
11. Faccone D. et al.: Molecular characterization of a clinical Haemophilus parainfluenzae isolate with cefotaxime resistance and decreased susceptibility to fluoroquinolones. Infect. Genet. Evol., 44, 2016.

12. Frickmann H. et al.: Difficulties in species identification within the genus Haemophilus - A pilot study addressing a significant problem for routine diagnostics. Eur. J. Microbiol. Immunol. (Bp)., 2, 4, 2014.

13. Frickmann H. et al.: Rapid discrimination of Haemophilus influenzae, $H$. parainfluenzae, and $H$. haemolyticus by fluorescence in situ hybridization (FISH) and two matrix-assisted laser-desorptionionization time-of-flight mass spectrometry (MALDI-TOF-MS) platforms. PLOS One, 4, 8, 2013.

14. Garcia-Vallvé S., Palau J, Romeu A.: Horizontal gene transfer in glycosyl hydrolases inferred from codon usage in Escherichia coli and Bacillus subtilis. Mol. Biol. Evol., 16, 9, 1999.

15. Giufrè M. et al.: Carriage of Haemophilus influenzae in the oropharynx of young children and molecular epidemiology of the isolates after fifteen years of $H$. influenzae type b vaccination in Italy. Vaccine, 46, 33, 2015.

16. Harper J. J., Tilse M. H.: Biotypes of Haemophilus influenzae that are associated with noninvasive infections. J. Clin. Microbiol., 11, 29, 1991.

17. Hong M. J., Kim Y. D., Ham H. D.: Acute septic arthritis of the acromioclavicular joint caused by Haemophilus parainfluenzae: a rare causative origin. Clin. Rheumatol., 4, 34, 2015.

18. Kaushik M. et al.: Case report of Haemophilus parainfluenzae sepsis in a newborn infant following water birth and a review of literature. AJP Rep., 2, 5, 2015.

19. Kilian M. Haemophilus (2003) In: Manual of clinical microbiology, 9th ed. Murray P. R., Baron E. J., Jorgensen J. H., Landry M. L., Pfaller M. A. (editors). Washington, DC: ASM Press; p. 636-648.

20. Kilian M.: A taxonomic study of the genus Haemophilus, with the proposal of a new species. J. Gen. Microbiol., 1, 93, 1976.

21. Koshkelashvili N. et al.: Polymicrobial infective endocarditis caused by Neisseria sicca and Haemophilus parainfluenzae. IDCases., 4, 2015.

22. Kosikowska U. et al.: The association of chronic hepatitis $\mathrm{C}$ with respiratory microbiota disturbance on the basis of decreased Haemophilus spp. colonization. Med. Sci. Monit., 22, 2016.

23. Kosikowska U. et al.: Nasopharyngeal and adenoid colonization by Haemophilus influenzae and Haemophilus parainfluenzae in children undergoin adenoidectomy and the ability of bacterial isolates to biofilm production. Medicine, 94, 2015.

24. Kosikowska U. et al.: Changes in the prevalence and biofilm formation of Haemophilus influenzae and Haemophilus parainfluenzae from the respiratory microbiota of patients with sarcoidosis. BMC Infect. Dis., 449, 16, 2016.

25. Kosikowska U. et al.: Haemophilus parainfluenzae as a marker of the upper respiratory tract microbiota changes under the influence of preoperative prophylaxis with or without postoperative treatment in patients with lung cancer. BMC Microbiol., 16, 2016.

26. Kosikowska U. et al.: Application of MALDI-TOF MS for identification of clinical isolates of bacteria from humans and animals. Diag. Lab., 1, 51, 2015.

27. Kosikowska U. et al.: Drug susceptibility of Haemophilus sp. and Staphylococcus aureus strains colonizing respiratory tract in children with mucoviscidosis. Przegl. Epidemiol., 2, 61, 2007.

28. Książczyk M. et al.: Application of routine diagnostic procedure, VITEK 2 Compact, MALDI-TOF MS, and PCR assays in adentification procedure of bacterial strain with ambiguous phenotype. Curr. Microbiol., 5, 72, 2016.

29. Kuhnert P. et al.: Identification of animal Pasteurellaceae by MALDITOF mass spectrometry. J. Microbiol. Methods, 89, 2012.

30. Mackenzie G. A. et al.: Epidemiology of nasopharyngeal carriage of respiratory bacterial pathogens in children and adults: crosssectional surveys in a population with high rates of pneumococcal disease. BMC Infect. Dis. 10, 2010.

31. Megraud F., Albouy C., Latrille J.: Haemophilus infections in pediatrics. Characterization of strains by biotype, serotype and the production of beta-lastamase. Pathol. Biol., 2, 31, 1983.

32. Middleton A. M. et al.: Haemophilus parainfluenzae infection of respiratory mucosa. Respir. Med., 97, 2003.
33. Munson E. L., Doern G. V.: Comparison of three commercial test systems for biotyping Haemophilus influenzae and Haemophilus parainfluenzae. J. Clin. Microbiol., 12, 45, 2007.

34. Musher D. M. (1996). Haemophilus Species. In: Source Medical Microbiology. 4th edition. Baron S. (editor). Galveston (TX): University of Texas Medical Branch at Galveston; Chapter in a book: 30 .

35. Nørskov-Lauritsen N.: Classification, identification, and clinical significance of Haemophilus and Aggregatibacter species with host specificity for humans. Clin. Microbiol. Rev., 2, 27, 2014.

36. Nørskov-Lauritsen N., Kilian M.: Reclassification of Actinobacillus actinomycetemcomitans, Haemophilus aphrophilus, Haemophilus paraphrophilus and Haemophilus segnis as Aggregatibacter actinomycetemcomitans gen. nov., comb. nov., Aggregatibacter aphrophilus comb. nov. and Aggregatibacter segnis comb. nov., and emended description of Aggregatibacter aphrophilus to include V factor-dependent and V factor-independent isolates. Int. J. Syst. Evol. Microbiol., 9, 56, 2006.

37. Oberhofer T. R., Back A. E.: Biotypes of Haemophilus encountered in clinical laboratories. J. Clin. Microbiol., 2, 10, 1979.

38. Oehler E., Valour F.: Septic osteoarthritis due to Haemophilus parainfluenzae. Med. Mal. Infect., 1, 44, 2014.

39. Page R. D. M.:. TREEVIEW: An application to display phylogenetic trees on personal computers. Comput. Appl. Biosci., 12, 1996.

40. Pan H. et al.: Nasal carriage of common bacterial pathogens among healthy kindergarten children in Chaoshan region, southern China: a cross-sectional study. BMC Pediatrics. 16, 2016.

41. Pillai A. et al.: A case of Haemophilus parainfluenzae pneumonia. Thorax, 55, 2000.

42. Pollard et al.: Structurural characterization of Haemophilus parainfluenzae lipooligosaccharide and elucidation of its role in adherence using an outer core mutant. Can. J. Microbiol., 54, 11, 2008.

43. Quentin R. et al.: Typing of urogenital, maternal, and neonatal isolates of Haemophilus influenzae and Haemophilus parainfluenzae in correlation with clinical source of isolation and evidence for a genital specificity of $H$. influenzae biotype IV. J. Clin. Microbiol., 27, 10, 1989.

44. Rayner M.G. et al.: Evidence of bacterial metabolic activity in culture-negative otitis media with effusion. JAMA, 279, 4, 1998.

45. Revest M. et al.: HACEK endocarditis: state-of-the-art. Expert Rev. Anti. Infect. Ther., 5, 15, 2016.

46. Sharara S. L. et al.: HACEK endocarditis: a review. Expert Rev. Anti. Infect. Ther., 6, 14, 2016.

47. Sottnek F. O., Albritton W. L.: Haemophilus influenzae biotype VIII. J. Clin. Microbiol., 4, 20, 1984.

48. Sulikowska A. et al.: Characteristics of Streptococcus pneumoniae, Haemophilus influenzae, and Moraxella catarrhalis isolated from the nasopharynges of asymptomatic children and molecular analysis of S. pneumoniae and H. influenzae strain replacement in the nasopharynx. J. Clin. Microbiol., 9, 42, 2004.

49. Takala A. K. et al.: Reduction of oropharyngeal carriage of Haemophilus influenzae type b (Hib) in children immunized with an Hib conjugate vaccine. J. Infect. Dis., 5, 164, 1991.

50. Taylor D. C. et al.: Biotypes of Haemophilus parainfluenzae from the respiratory secretions in chronic bronchitis. J. Med. Microbiol., 4, 36, 1992.

51. Tiller F.W., Kleinemas U.: Beta-lactamase producing haemophili occurrence in clinical materials and carriage in healthy children. J. Hyg. Epidemiol. Microbiol. Immunol., 30, 2,1986.

52. Uraz G., Simşek H., Celik B.: Beta-lactamase activities and resistance to antibiotics of Haemophilus influenzae, H. parainfluenzae and $H$. aphrophilus strains identified in throat cultures from children. Drug Metabol. Drug Interact., 3, 16, 2000.

53. Wallace R. J. et al.: Nontypable Haemophilus influenzae (biotype 4) as a neonatal, maternal, and genital pathogen. Rev. Infect. Dis., $5,1,1983$.

54. Watson K. C., Kerr E. J., Hinks C. A.: Distribution of biotypes of Haemophilus influenzae and H. parainfluenzae in patients with cystic fibrosis. J. Clin. Pathol., 7, 38, 1985.

55. Yew H. S. et al.: Association between HACEK bacteraemia and endocarditis. J. Med. Microbiol., 6, 63, 2014. 